\title{
Exenatide improves glucocorticoid-induced glucose intolerance in mice
}

This article was published in the following Dove Press journal:

Diabetes, Metabolic Syndrome and Obesity: Targets and Therapy

25 January 2011

Number of times this article has been viewed

\author{
Ruiying Zhao ${ }^{1,2^{*}}$ \\ Enrique Fuentes-Mattei ${ }^{1,2^{*}}$ \\ Guermarie Velazquez- \\ Torres ${ }^{1,3}$ \\ Chun-Hui Su ${ }^{1,2}$ \\ Jian Chen' \\ Mong-Hong Lee ${ }^{1,2}$ \\ Sai-Ching Jim Yeung ${ }^{4,5}$ \\ 'Department of Molecular and \\ Cellular Oncology, University of \\ Texas MD Anderson Cancer Center, \\ Houston, TX, USA; ${ }^{2}$ Program in \\ Genes and Development, ${ }^{3}$ Program \\ in Cancer Biology, Graduate \\ School of Biomedical Sciences, \\ University of Texas Health Science \\ Center in Houston, Houston, TX, \\ USA; ${ }^{4}$ Department of Endocrine \\ Neoplasia and Hormonal Disorders, \\ ${ }^{5}$ Department of Emergency Medicine, \\ University of Texas MD Anderson \\ Cancer Center, Houston, TX, USA \\ *Both authors contributed equally.
}

Correspondence: Sai-Ching Jim Yeung UTMD Anderson Cancer Center, I5I 5 Holcombe Blvd, Unit I468, Houston, Texas 77030, USA

$\mathrm{Tel}+\mathrm{I} 7137454516$

Fax + I 713563 449|

Email syeung@mdanderson.org
Abstract: Exenatide is an incretin mimetic that is recently available in the US for the treatment of diabetes. There is a paucity of information on the effects of exenatide in glucocorticoid (GC)-induced diabetes. Although the effect of continuous intravenous infusion of exenatide on GC-induced glucose intolerance has been investigated before in healthy human males receiving oral prednisolone, we investigated the efficacy of a single subcutaneous dose of exenatide $(3 \mu \mathrm{g} / \mathrm{kg})$ in lowering blood glucose in GC-induced glucose intolerance in C57BL/6 mice. In a longitudinal experiment, the area under the curve (AUC) of oral glucose tolerance tests (OGTT) significantly increased after dexamethasone $(P=0.004)$, which was subsequently decreased by exenatide $(P<0.001)$. A cross-sectional experiment showed that exenatide improved glucose tolerance compared with placebo in a mouse model of dexamethasone-induced glucose intolerance. AUC of OGTT in the exenatide group were significantly $(P<0.001)$ lower than in the placebo group. Insulin tolerance tests (ITT) demonstrated that exenatide decreased the ability of the mice to tolerate insulin compared with placebo. The AUC of ITT in the exenatide group were also significantly $(P=0.006)$ lower than in the placebo group. In conclusion, a single dose of exenatide was able to decrease glucose intolerance and insulin resistance in these placebocontrolled experiments. Future clinical trials are justified to investigate the role of exenatide in the treatment of GC-induced glucose intolerance/diabetes.

Keywords: exenatide, dexamethasone, glucocorticoid, insulin resistance, mouse model

Glucocorticoids (GC) induce hyperglycemia, ${ }^{1}$ and hyperglycemia have an adverse effect on the outcome of chemotherapy for acute lymphocytic leukemia (ALL). ${ }^{2}$ Patients with hyperglycemia ( $\geq 200 \mathrm{mg} / \mathrm{dL}$ ) during induction chemotherapy with the hyper-CVAD regimen (fractionated cyclophosphamide, vincristine, doxorubicin, and dexamethasone alternating with high-dose methotrexate and cytarabine) were found to have shorter complete remission durations than those with normal glucose. ${ }^{2}$ The patients had hyper-CVAD, which included dexamethasone $40 \mathrm{mg}$ on days 1-4 and days 11-14 in odd-number courses (courses 1, 3, 5, and 7) and methylprednisolone $50 \mathrm{mg}$ twice daily on days 1-3 in even-number courses (courses 2, 4, 6, and 8). Antidiabetic medications such as thiazolidinediones (eg, pioglitazone) ${ }^{3,4}$ and biguanides (metformin) ${ }^{3}$ can be used to improve the insulin resistance induced by GC. Insulin has been commonly used to manage acute exacerbation in hyperglycemia induced by glucocorticoids in most clinical settings. ${ }^{5}$ However, insulin has a promoting effect on growth and chemoresistance to daunorubicin of leukemic cell lines and primary samples of ALL (Yeung, unpublished data); therefore, a rapidly effective therapy without a malignancy-promoting effect needs to be identified for the treatment of GC-induced hyperglycemia in ALL 
patients undergoing chemotherapy before medications such as metformin and pioglitazone can exert their full antidiabetic effect or when oral antidiabetic medications are unable to control hyperglycemia satisfactorily. Exenatide is a glucagonlike peptide- 1 mimetic recently approved for the treatment of diabetes. Our recent investigation did not find any direct promoting effect of exenatide on cancer cells. ${ }^{6}$ Its effectiveness for GC-induced hyperglycemia is unknown. Therefore, we tested the effectiveness of exenatide in controlling GC-induced hyperglycemia in a mouse model.

We induced glucose intolerance by intraperitoneal (IP) injection of dexamethasone (dexamethasone sodium phosphate for injection, APP Pharmaceuticals, Raleigh, $\mathrm{NC}$, USA; $4 \mathrm{mg} / \mathrm{mL}$ ) into adult C57BL6 mice (males and females). Blood glucose levels were measured using a FreeStyle Glucose Meter (Abbott Laboratories, Abbott Park, IL, USA). Blood samples were collected by cutting into the soft tissue of the distal 2-mm portion of the mice's sterilized tails using a scalpel and gentle squeezing to obtain two or more drops of blood. The first drop was discarded; the second drop was applied on the test strip. Additional blood (up to $60 \mu \mathrm{L}$ ) was collected by submandibular bleeding (superficial temporal vein) for hormone analysis when indicated. An oral glucose tolerance test (OGTT) was performed after a 15- to 18-hour fast. Blood glucose levels were measured immediately prior to glucose administration ( $0 \mathrm{~min})$ and at 15,30 , 60 , and $120 \mathrm{~min}$ after glucose $(1.5 \mathrm{~g} / \mathrm{kg}$ as a $50 \% \mathrm{w} / \mathrm{v}$ solution in water) administration by orogastric gavage. An insulin tolerance test (ITT) was performed after a 6-hour fast. Blood glucose levels were measured immediately prior to insulin administration ( $0 \mathrm{~min}$ ) and at 15, 30, 60, and $120 \mathrm{~min}$ after recombinant human insulin $(0.75 \mathrm{IU} / \mathrm{kg})$ IP administration.

Area under the curve (AUC) for OGTT and ITT from each animal were calculated by interpolation and integration. Changes in AUC or in hormone levels, where appropriate, were compared. For comparisons between the two groups, Student's $t$-test or Mann-Whitney rank-sum test were used, where appropriate, based on the normality of distribution and equality of variances.

Using the mouse model of diabetes induced by dexamethasone $(20 \mathrm{mg} / \mathrm{kg} /$ day IP every morning for 5 days), a longitudinal experiment was performed. OGTT were performed in nine 8- to 10 -week-old C57BL/6 mice at baseline and after dexamethasone injection on day 4 . After dexamethasone injection on day 5 , the mice were injected with exenatide (Lilly, Indianapolis, IN, USA; $250 \mu \mathrm{g} / \mathrm{mL}$, diluted in sterile normal saline to appropriate volume just before use) $3 \mu \mathrm{g} / \mathrm{kg}$ subcutaneously (SQ) 1 hour prior to the OGTT. The average blood glucose levels during the OGTT increased at most of the time points from baseline to day 4 after dexamethasone administration, and decreased at most of the time points from baseline and day 4 to day 5 after dexamethasone and exenatide administration (Figure 1A). The average change in AUC between the first and second OGTT of each mouse was significantly higher than 0 (one sample $t$-test, $P=0.004)$; the average change in AUC between the second

A
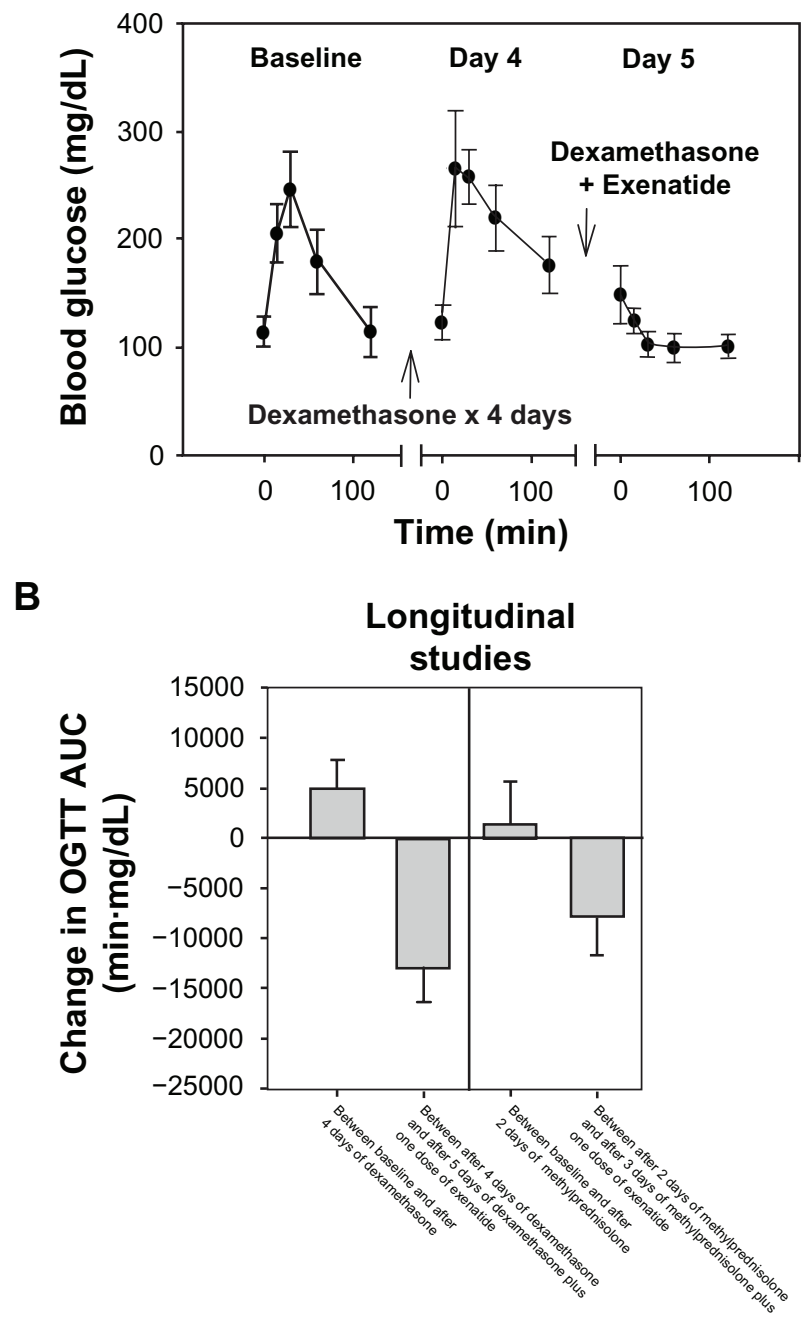

Figure I Exenatide suppressed the rise in blood glucose after an oral glucose load in mice with glucocorticoid-induced glucose intolerance. A) Average blood glucose levels of nine adult C57BL/6 mice during an OGTT performed on three occasions are plotted against time. The error bars represent $95 \% \mathrm{Cl}$. After baseline OGTT, each mouse was injected dexamethasone $(20 \mathrm{mg} / \mathrm{kg} / \mathrm{day})$ IP for 4 days. The day 4 OGTT was performed several hours after dexamethasone injection in the morning. On day 5, the OGTT was performed after injection of dexamethasone IP and exenatide $(3 \mu \mathrm{g} / \mathrm{kg})$ SQ. B) The average change in AUC between the first and second OGTT of each mouse and between the second and third OGTT are plotted in the left panel for the longitudinal experiment described in A. Similar data for a separate experiment using a less potent glucocorticoid (methylprednisolone) and shorter duration than in A) are plotted in the right panel. The error bars represent $95 \% \mathrm{Cl}$.

Abbreviations: AUC, area under the curve; $\mathrm{Cl}$, confidence intervals; IP, intraperitoneal; OGTT, oral glucose tolerance test. 
and third OGTT was significantly lower than 0 (one sample $t$-test, $P<0.001$ ) (Figure 1B). Therefore, dexamethasone, given in the manner described, increased glucose intolerance in C57BL/6 mice, and a single SQ injection of exenatide ( $3 \mu \mathrm{g} / \mathrm{kg}$ ) significantly suppressed the rise in blood glucose after the oral glucose load.

In a second longitudinal experiment, ten 8- to 10-weekold C57BL/6 mice were injected with methylprednisolone (methylprednisolone sodium succinate for injection, Pfizer, New York, NY, USA; $40 \mathrm{mg} / \mathrm{mL}$ diluted to $4 \mathrm{mg} / \mathrm{mL}$ in normal saline) $20 \mathrm{mg} / \mathrm{kg} /$ day IP every morning for 3 days instead of dexamethasone. The lower potency of methylprednisolone and shorter duration of glucocorticoid treatment than in the first experiment was intended to test whether exenatide would induce hypoglycemia when GC-induced glucose intolerance was mild. OGTT were performed at baseline, day 2, and day 3, with the exenatide given 1 hour prior to the OGTT on day 3. The average change in AUC between the first and second OGTT of each mouse was not significantly higher than 0 (one sample $t$-test, $P=0.476$ ); the average change in AUC between the second and third OGTT was significantly lower than 0 (one sample $t$-test, $P=0.001$ ) (Figure 1B). Although a single dose of exenatide significantly reduced the AUC in the methylprednisolone-treated mice, the glucose levels were stable within the normal range for the duration of the OGTT, and no hypoglycemia (defined as blood glucose $<80 \mathrm{mg} / \mathrm{dL}$ ) was observed.

To corroborate the effect of a single dose of exenatide on GC-induced glucose intolerance, a cross-sectional, placebocontrolled experiment was performed using the mouse model of diabetes induced by dexamethasone $(20 \mathrm{mg} / \mathrm{kg} /$ day for 5 days $)$. Exenatide ( $3 \mu \mathrm{g} / \mathrm{kg}$ SQ 1 hour prior to OGTT) improved glucose tolerance (Figure 2A) compared with a placebo control group (Figure 2A). Using the same mouse model, an ITT demonstrated that exenatide (Figure 2B) decreased the ability of the mice to tolerate insulin compared with placebo (Figure 2B). AUC of OGTT and ITT for the exenatide group and the control group are shown in Figure 2C. In the OGTT, the mean AUC of the exenatide-treated mice $(\mathrm{n}=18)$ was significantly ( $P<0.001$, Mann-Whitney rank-sum test) lower than that of the placebo-treated mice $(n=17)$. In the ITT, the mean AUC of the exenatide-treated mice $(n=9)$ was also significantly ( $P=0.006$, Mann-Whitney rank-sum test) lower than that of the placebo-treated mice $(\mathrm{n}=13)$. Therefore, a single dose of exenatide was able to decrease glucose intolerance and insulin resistance in these placebo-controlled experiments.

Extra blood samples were collected at time 0 (baseline) and 30 min after glucose administration during the OGTT in a subset of animals ( $\mathrm{n}=5$ each for exenatide and control groups) for hormone analyses. A total volume of $20 \mu \mathrm{L}$ of plasma from each time point was assayed in duplicate in a multiplex immunodetection analysis for amylin, C-peptide, ghrelin, gastric inhibitory polypeptide, glucagon-like peptide-1, interleukin-6, insulin, glucagon, resistin, leptin, monocyte chemotactic protein-1, pancreatic polypeptide, polypeptide $Y Y$, and tissue necrosis factor- $\alpha$ (Milliplex Mouse Metabolic Hormone Magnetic Bead Panel, Millipore, Billerica, MA, USA). Amylin, tissue necrosis factor- $\alpha$, monocyte chemotactic protein-1, pancreatic polypeptide, polypeptide YY, glucagon, glucagonlike peptide-1, and ghrelin were below the detection limits. Gastric inhibitory polypeptide, interleukin-6, and resistin did not show any consistent changes between baseline (time 0 ) and 30 min after oral glucose load. The changes between the two time points $\left(\Delta\right.$ Hormone $\left._{0-30 \mathrm{~min}}\right)$ in C-peptide, insulin, and leptin are plotted for the exenatide-treated group and the placebotreated control group in Figure 2D. Although there might be trends that exenatide decreased the change in C-peptide and insulin levels and that exenatide increased the rise in leptin level 30 min after the orogastric glucose load, the variances in the data were too large to reach any statistical conclusions.

Exenatide is highly unlikely to have a promoting effect on the growth of malignancies. Despite concerns based on the observation of benign c-cell thyroid adenomas in rats that received exenatide for 2 years, there is no evidence to support a link between exenatide and medullary thyroid cancer. Our published data show that exenatide does not have any promoting effects on the growth of breast cancer and pancreatic cancer cell lines in cell culture. ${ }^{6}$ Although exenatide enhances glucose-dependent insulin secretion in type 2 diabetic patients by activation of glucagon-like peptide-1 receptor, exenatide delays gastric emptying and reduces postprandial glucose absorption and peak plasma insulin level. ${ }^{7}$ There is cell culture experimental evidence to suggest that GC induce apoptosis in insulin-secreting cells and that exenatide can protect against the GC-induced apoptosis. ${ }^{8}$ The incretin effect is impaired after induction of reduced glucose tolerance and insulin resistance in healthy males by oral prednisolone treatment, a high-calorie diet, and physical inactivity. ${ }^{9}$ At the 2010 meeting of the American Diabetes Association, van Raalte et al reported that exenatide prevented glucose intolerance as assessed during a standardized meal test. ${ }^{10}$ Our mouse model data have also shown that exenatide is effective in reversing GC-induced glucose intolerance. As shown by hyperglycemic clamping, exenatide improves $\beta$-cell function in humans treated with prednisolone. ${ }^{10}$ Our ITT data suggest that exenatide may also be acting on the peripheral tissues and improving their 
A

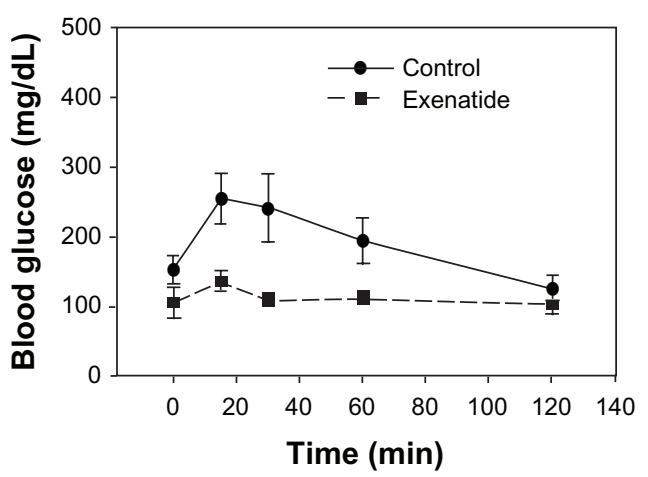

C

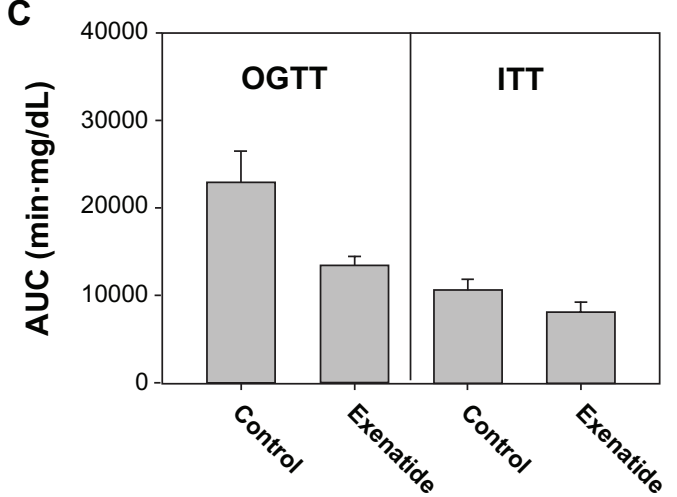

B

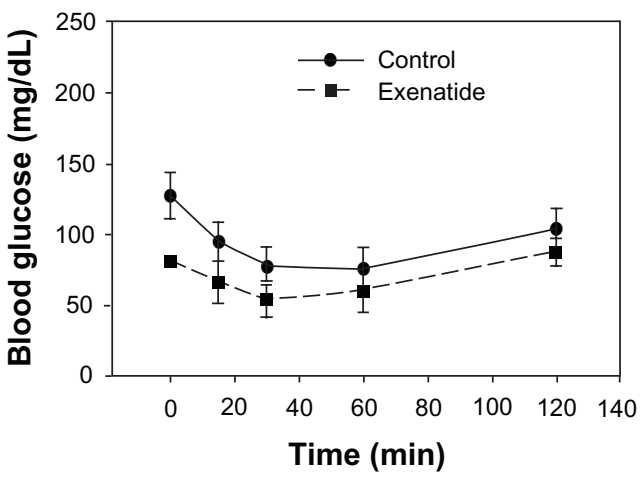

D

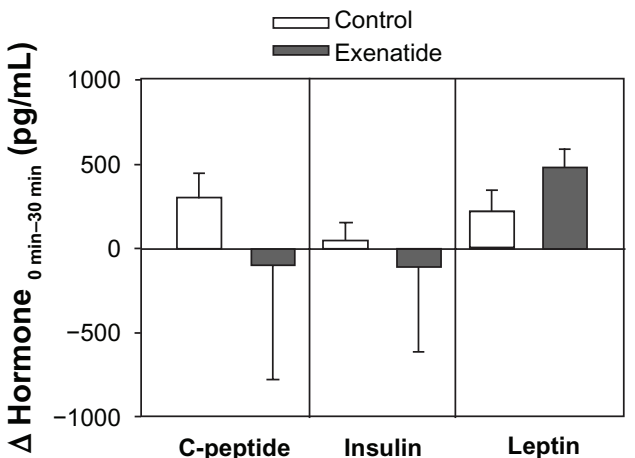

Figure 2 Exenatide suppressed the rise in blood glucose after an oral glucose load and decreased insulin tolerance in dexamethasone-treated mice compared with placebo. A) Using the mouse model of diabetes induced by dexamethasone $(20 \mathrm{mg} / \mathrm{kg} /$ day for 5 days $)$, the cross-sectional comparison of OGTT between an exenatide-treated group (squares) and a placebo-treated control group (circles) is shown. Error bars - 95\% Cl. B) Using the same mouse model, the cross-sectional comparison of ITT between an exenatide-treated group (squares) and a placebo-treated control group (circles) is shown. Error bars - 95\% CI. C) AUC for OGTT in A and ITT in B were plotted for the exenatide and control groups as labeled. Error bars - $95 \% \mathrm{Cl}$. D) Extra blood samples were collected at time 0 (baseline) and 30 min after glucose administration during OGTT in a subset of animals for hormone analyses. The changes in C-peptide, insulin, and leptin between the two time points were plotted for the exenatide-treated group (dark bars) and the placebo-treated control group (white bars). Error bars - standard errors.

Abbreviations: AUC, area under the curve; Cl, confidence intervals; IP, intraperitoneal; ITT, insulin tolerance test; OGTT, oral glucose tolerance test.

sensitivity to insulin. The trend in the hormone data associated with the OGTT in our study suggests that increasing insulin secretion may not be the primary mechanism for the efficacy of exenatide in lowering postprandial glucose. We speculate that delay in gastric emptying, resulting in delayed or decreased absorption of glucose, may also play a major role. Further study, including parenteral glucose tolerance tests, long-term observations, and/or evaluation of peripheral sensitivity to insulin by glucose clamping, may further elucidate the mechanisms involved in the beneficial effect of exenatide in GC-induced glucose intolerance.

Because human data ${ }^{10}$ and our mouse data have shown that exenatide is effective in reversing GC-induced glucose intolerance, exenatide may be a more appropriate agent than exogenous insulin or insulin secretagogues in the management of acute exacerbations of hyperglycemia in cancer patients, especially in the clinical scenario of GCinduced hyperglycemia encountered in the treatment of ALL patients. Clinical trials are justified to evaluate the effectiveness of exenatide for glucose control in GC-induced glucose intolerance and whether exenatide can reverse the decrease in complete remission duration and survival in ALL patients with steroid-induced hyperglycemia.

\section{Acknowledgments}

We acknowledge the skilled technical assistance of Yi Qiao. This study was partially supported by grants from the following agencies: the University of Texas MD Anderson Cancer Center, Division of Internal Medicine Multidisciplinary Research Program (PI: SC Yeung); US National Institute of Health (NIHRO1CA 089266, PI: MH Lee); Department of Defense, Breast Cancer Research Program of the Office of the Congressionally Directed Medical Research Programs (Synergistic Idea Development Award BC062166, PI: SC Yeung and MH Lee); and Susan G Komen Foundation for Breast Cancer Research (Promise grant KG081048, PI: SC Yeung). This research was also supported in part by a cancer prevention fellowship for $\mathrm{G}$ Velazquez-Torres from 
NIH-NCI (R25TCA57730; PI: S Chang) and by the Training Grant Program in Molecular Genetics for E Fuentes-Mattei from NIH-NCI (2T32CA009299-31A1; PI: G Lozano). The funding bodies had no role in study design; in the collection, analysis, and interpretation of data; in the writing of the manuscript; or in the decision to submit the manuscript for publication.

\section{Disclosure}

No conflicts of interest were declared in relation to this paper.

\section{References}

1. Van Raalte DH, Ouwens DM, Diamant M. Novel insights into glucocorticoid-mediated diabetogenic effects: towards expansion of therapeutic options? Eur J Clin Invest. 2009;39:81-93.

2. Weiser MA, Cabanillas ME, Konopleva M, et al. Relation between the duration of remission and hyperglycemia during induction chemotherapy for acute lymphocytic leukemia with a hyperfractionated cyclophosphamide, vincristine, doxorubicin, and dexamethasone/methotrexatecytarabine regimen. Cancer. 2004;100:1179-1185.
3. Hoogwerf B, Danese RD. Drug selection and the management of corticosteroid-related diabetes mellitus. Rheum Dis Clin North Am. 1999;25:489-505.

4. Willi SM, Kennedy A, Brant BP, Wallace P, Rogers NL, Garvey WT. Effective use of thiazolidinediones for the treatment of glucocorticoidinduced diabetes. Diabetes Res Clin Pract. 2002;58:87-96.

5. Clore JN, Thurby-Hay L. Glucocorticoid-induced hyperglycemia. Endocr Pract. 2009;15:469-474.

6. Feng YH, Velazquez-Torres G, Gully C, Chen J, Lee MH, Yeung SC. The impact of type 2 diabetes and antidiabetic drugs on cancer cell growth. J Cell Mol Med. 2010 May 3. [Epub ahead of print].

7. Linnebjerg H, Park S, Kothare PA, et al. Effect of exenatide on gastric emptying and relationship to postprandial glycemia in type 2 diabetes. Regul Pept. 2008;151(1-3):123-129.

8. Ranta F, Avram D, Berchtold S, et al. Dexamethasone induces cell death in insulin-secreting cells, an effect reversed by exendin-4. Diabetes. 2006;55:1380-1390.

9. Hansen KB, Vilsboll T, Bagger J, Holst JJ, Knop FK. Reduced glucose tolerance and insulin resistance induced by steroid treatment, relative physical inactivity, and high-calorie diet impairs the incretin effect in healthy subjects. J Clin Endocrinol Metab. 2010;95:3309-3317.

10. Van Raalte DH, van Genugten RE, Linssen MML, Ouwens MDM, Diamant $\mathrm{M}$. The glucagon-like peptide receptor agonist exenatide protects against glucocorticoid-induced glucose intolerance and islet-cell dysfunction in humans. Diabetes. 2010;59(Suppl 1):A62.

\section{Publish your work in this journal}

Diabetes, Metabolic Syndrome and Obesity: Targets and Therapy is an international, peer-reviewed open-access journal committed to the rapid publication of the latest laboratory and clinical findings in the fields of diabetes, metabolic syndrome and obesity research. Original research, review, case reports, hypothesis formation, expert opinion and commentaries are all considered for publication. The manuscript management system is completely online and includes a very quick and fair peer-review system, which is all easy to use. Visit http://www.dovepress.com/testimonials.php to read real quotes from published authors. 